opinions acquises à la longue et qui passent pour des vérités, méconnaître la réalité et conseiller des pratiques dont l'agriculture ne peut, tout bien pesé, retirer le moindre bénéfice. Dans le cas présent, la castration, que l'on a tant recommandée à bien des points de vue, n'est recommandable, comme l'a dit LERMat, que pour les vaches qui ne doivent plus, ou ne peuvent plus " porter ".

\title{
RECHERCHES SUR LA CROISSANCE DES MAMMIFÈRES. - SES RELATIONS AVEC LA COMPOSITION CHIMIQUE DU LAIT DE LA MÈRE
}

\author{
par G. BELLE, \\ Docteur-Vétérinaire.
}

(Suite).

Ce que devient 1 kilogramme d'animal au bout de trente jours.Si l'on considère ce que devient 1 kilog. d'animal au bout de 30 jours, on a les résultats suivants :

\begin{tabular}{|c|c|c|c|}
\hline & ilog de lapin & devient & $12 \mathrm{~kg} .000$ \\
\hline & " de chien & " & $6 \mathrm{~kg} .000$ \\
\hline & " de porc & $n$ & $5 \mathrm{~kg} \cdot 700$ \\
\hline & de cobaye & " & $3 \mathrm{~kg} .100$ \\
\hline & " de chat & " & $3 \mathrm{~kg} .000$ \\
\hline & d'agneau & * & $2 \mathrm{~kg} \cdot 750$ \\
\hline & de chevreau & $"$ & $2 \mathrm{~kg} .300$ \\
\hline & " de poulain & " & $1 \mathrm{~kg} .750$ \\
\hline & " de veau & $"$ & $1 \mathrm{~kg} .680$ \\
\hline & " d'enfant & , & $1 \mathrm{~kg} .250$ \\
\hline
\end{tabular}

C'est done le lapin qui croît le plus rapidement, et l'enfant qui vient en tout dernier lieu.

Le lait et I'alimentation du jeune. - Le lait est l'aliment indis pensable à la vie du nouveau-né ; lui seul peut être assimilé par un organisme aussi fragile. C'est un aliment complet : nous verrons plus loin qu'il contient tous les principes nécessaires à la vie et à la croissance.

Mais si tous les mammifères, à quelque espèce qu'ils a ppartiennent, ont un besoin indispensable du lait, tous ne l'utilisent pas dans les mêmes proportions. Si l'on considère les animaux domestiques et l'enfant, on est frappé de ce fait que certains jeunes (en particulier ceux naissant complètement nus ou peu couverts de poils) doivent recourir à la sécrétion lactée de leur mère, d'extrême urgence et pour un temps plus long que les autres, venant au monde vêtus et qui seront capables de puiser eux-mêmes bien vite dans le milieu extérieur leur nourriture. De ce point de vue, le Professeur Ch. Porcher a 
PL. XV.

CE QUE DEVIENT AU BOUT DE 30 JOURS 1 KG. POIDS A LA NAISSANCE

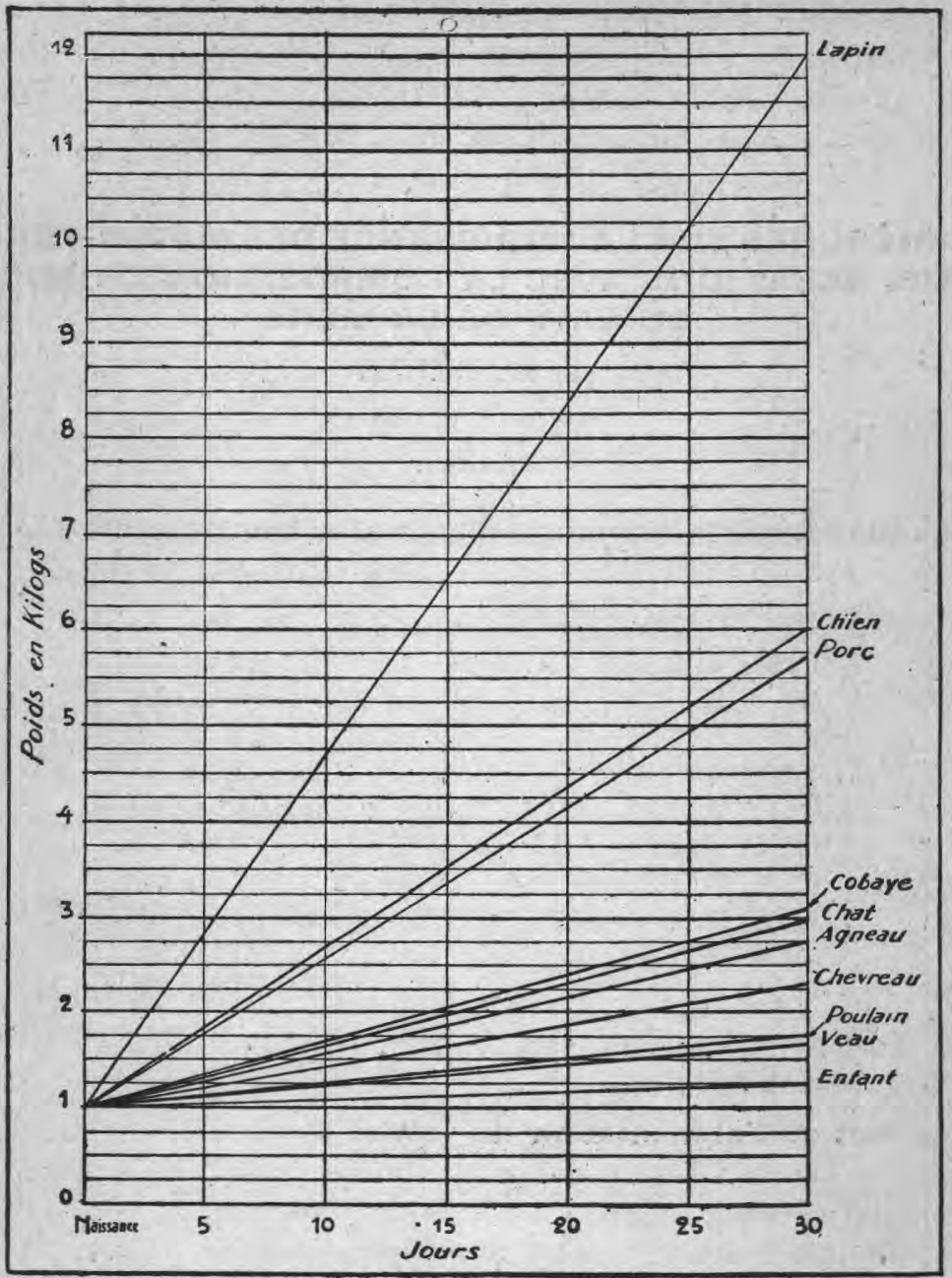

établi une opposition très marquée entre l'enfant, le lapereau, le chiot, le chaton, le rat et la souris d'une part, le veau, le poulain, l'agneau d'autre part, et enfin le cobaye. Nous laisserons ici de côté l'enfant, qui a bénéficié de l'intelligence humaine et qui, du fait de la civilisation, n'a pas une existence comparable à celle des animaux.

Si nous comparons le chien, le chat, le lapin, le cobaye dont la durée moyenne de la vie est de 10 ans environ, nous constatons que plus la vie intra-utérine est longue, plus la sécrétion mammaire se restreint. A ce sujet, la comparaison du la pin et du cobaye est clas- 
CE QUE DEVIENT A LAGE ADULTE 1 KG. DE POIDS A LA NAISSANGE

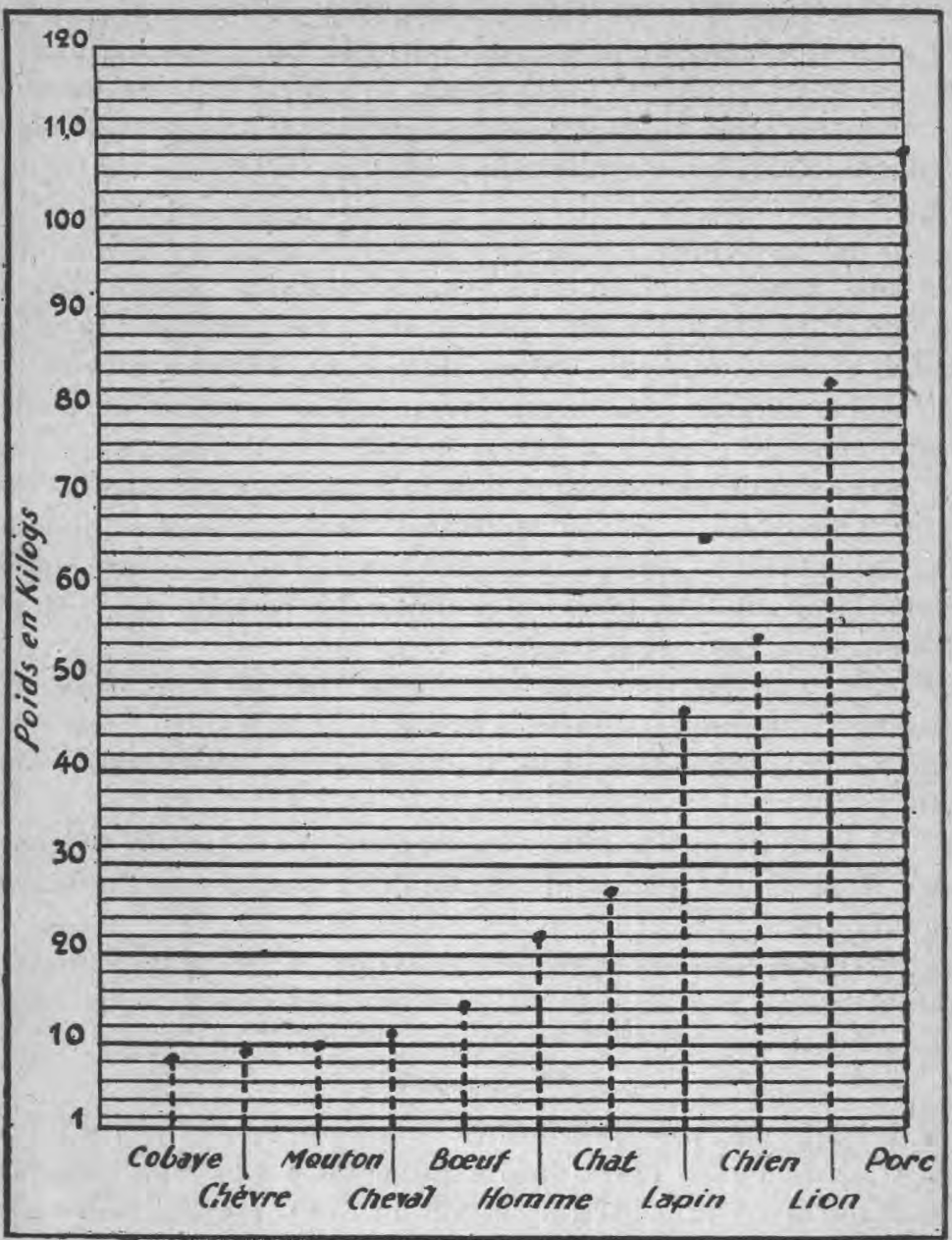

sique. La durée moyenne de la gestation est chez le cobaye de 60 à 65 jours en moyenne (comme chez la chienne par conséquent). Le jeune qui, à la naissance, pèse 95 grammes en moyenne, soit le $1 / 8^{\mathrm{e}} \mathrm{du}$ poids de l'adulte, a le corps couvert de poils, l'œil ouvert et vif ; il peut trotter et se passer de sa mère. Cette constatation, faite depuis longtemps déjà par le professeur $\mathrm{Ch}$. Porcher, a été traduite par $\mathrm{Ch}$. Richet d'une façon très heureuse : le cobaye naît adulte. De ce fait, le nouveau-né, resté longtemps dans le sein, de sa mère, ne tète que pendant quelques jours (une semaine à peine) et, bien vite, il est ca pable de trouver tout seul sa subsistance dans le milieu extérieur.

La lapine porte pendant 30 jours environ; elle met bas 5,6 petits et 
da vantage, qui viennent au monde nus et a veugles; leur poids (100 gr. en moyenne) est très voisin de celui du cobaye, mais pour arriver à l'état adulte, le la pereau devra s'accroître 47 fois environ', et non plus 8 fois comme le cobaye. - Le lapin naît done dans des conditions bien moins fa vorables que le jeune cobaye ; avant de pouvoir manger seul, jl a besoin de téter pendant 28 à 30 jours; le lait de sa mère lui est indis pensable.

La durée moyenne de la gestation chez la chatte et la chienne est à peu près identique (55-56 jours pour la première ; $63-65$ jours pour la seconde). Le chat et le chien naissent nus et a veugles; le premier pèse $150 \mathrm{gr}$, environ, le $2 \mathrm{e}, 365 \mathrm{gr}$. Mais ce qu'il faut constater, c'est que le poids du chat à sa naissance est le $1 / 26^{\mathrm{e}}$ environ du poids de l'adulte, celui du chien le $1 / 54^{\mathrm{e}}$ ou le $1 / 55^{\mathrm{e}}$. Le chien, toutes choses étant égales d'ailleurs, pèse donc à sa naissance deux fois moins que le chat. L'éta pe à franchir pour arriver à l'état adulte sera done plus longue pour le chien que pour le chat ; aussi l'alimentation lactée se pousuit-elle pendant 50 jours environ dans l'espèce canine et 40 jours seulement dans l'es pèce féline.

De ceci, nous pouvons conclure que la durée de la sécrétion lactée est inversement proportionnelle à la durée de la gestation, ceci parce qu'elle est la continuation et le complément de l'alimentation intrautérine.

Si l'on considère l'existence des mammifères depuis la conception jusqu'à la mort, on peut la diviser en deux périodes de durée nettement inégales :

La première s'étendant jusqu'au sevrage pendant laquelle la vie du jeune est fonction de celle de sa mère ; elle peut se subdiviser en deux cycles :

\section{Cycle mammaire}

Le premier ou eycle intra-utérin ;

Le second ou cycle extra-utérin, qui va de la naissance au sevrage.

Pendant lavie intra-utérine, la vie du fœtus est intimement liéee à celle de sa mère, puisqu'il puise en elle sa nourriture, qu'il déverse ses produits de désassimilation dans l'organisme maternel, puisqu'en un mot, il fait partie intégrante de son corps.

Après la naissance, les conditions de vie sont légèrement modifiées, puisqu'à partir de ce moment-là, le fœetus se trouve dans le milieu extérieur. Il pourrait à la rigueur vivre sans le concours de sa mère, grâce à l'allaitement artificiel, mais, en principe, sa mère lui est nécessaire et indispensable. C'est encore elle qui le nourrit en lui donnant le seul aliment que son organisme soit capable d'assimiler : le lait ; or ce lait, c'est encore un produit de sécrétion de l'organisme maternel que le jeune absorbe non plus par esmose à travers l'ectoderme placentaire, mais par la voie digestive. Bref, on peut dire que le 
nouveau-né ne peut pas se passer de sa mère durant les quelques mois qui suivent sa naissance.

La deuxième période de la vie commence au sevrage, e'est-à-dire à l'époque où le jeune trouve dans le milieu extérieur les principes nutritifs indis pensa bles à son développement, et elle s'étend jusqu'à la mort. Elle a done une durée beaucoup plus considérable que la précédente.

L'alimentation lactée serait donc la continuation sous une autre forme et dans un monde nouveau, au contact de l'air et de la lumière, au moment où l'être est complètement formé, de l'alimentation intrautérine.

L'enfant est un être à part dans l'échelle des mammifères. Il vient au monde extrêmement chétif et frêle, à la merci de toutes les circonstances extérieures ; il ne sait subvenir tout seul à ses besoins, il est sous la protection de sa mère ou de sa nourrice pendant 18 mois environ ; jusqu'à cet âge, il est incapable de trouver ailleurs que dans le lait sa nourriture. Mais l'enfant n'occupe-t-il pas une place privilégiée par rapport aux autres mammifères ? Il ne vit pas d'une vie que l'on pourrait qualifier de naturelle ; il est choyé et aussi longtemps que le lait lui est nécessaire, on le lui donne soit par l'allaitement naturel, soit par l'allaitement artificiel. Une alimentation-lactée d'une aussi longue durée est une conséquence de la civilisation. Autrefois, au début de l'existence du monde, et même bien plus tard, les enfants étaient accoutumés beaucoup plus vite à chercher dans le milieu extérieur leur nourriture. De nos jours, on rencontre, dans certaines familles pauvres, des enfants de 5 ou 6 mois qui mangent déjà des potages, et nous en a vons vu qui, à un an, étaient capables d'absorber quelques feuilles de laitue. Ces exemples ne sont pas heureusement une règle générale, et il est bien certain que si la mère n'était qu'une simple machine à fournir le lait (ce qui se passe chez l'animal), et qu'elle n'ait pas recours à l'allaitement artificiel, l'enfant serait sevré beaucoup plus tôt et serait obligé de trouver avant l'âge de 15 -18 mois un aliment ailleurs que dans le lait. Les principes de la civilisation actuelle n'ont pas voulu contraindre l'enfant à un exercice aussi rude ; il faut lui donner, par le lait, le maximum de résistance; il faut que son organisme soit fort avant d'être appelé à assimiler autre chose que du lait.

Done, au point de vue alimentation dans le jeune âge, l'enfant ne peut pas être comparé aux animaux. Il est dans des conditions nettement plus favorables qu'eux pour grandir.

Si l'on compare la durée de l'alimentation lactée (15 mois en moyenne) a vec la durée moyenne de la vie de l'homme (45 à 50 ans), on constate que le ra pport $\frac{\text { durée de l'alimentation lactée }}{\text { durée moyenne de la vie }}$ est égal à $\frac{1}{38}$ environ ; ce qui peut se traduire ainsi : 
Sur une existence de 38 jours, l'enfant tète 1 jour; or c'est chez l'homme que le rapport est le plus élevé, ainsi qu'en témoigne le tableau ci-dessous :

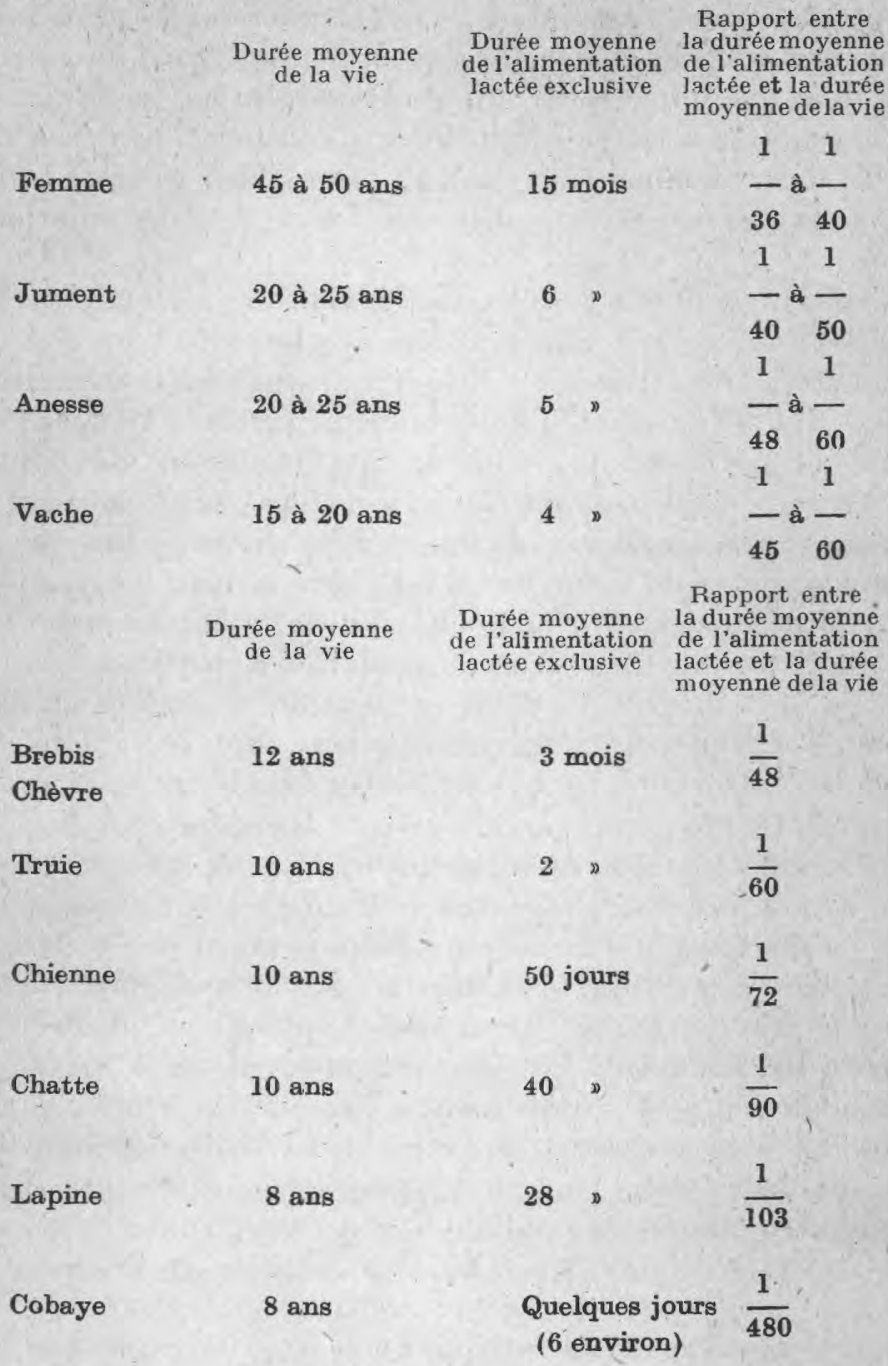

Certes, ce tableau n'est peut-être pas l'expression absolue de la vérité ; nous nous sommes efforcé de nous en rapprocher le plus possible. Il est difficile d'établir chez nos animaux domestiques une valeur pour la durée moyenne de la vie ; leur vie est fonction d'un grand nom'bre de facteurs dont les plus essentiels sont les soins a pportés à leur nourriture et au respect de leurs forces. De plus, en ce qui concerne les espèces chevaline, bovine, ovine, caprine, 
porcine, la fin habituelle est très souvent la boucherie ; le la pin, lui aussi, sert de nourriture à l'homme.

Ces animaux ont une existence qui se termine brutalement, et, si on les laissait mourir de leur propre mort, on serait frappé du nombre d'années qu'ils auraient encore à vivre.

Les chiffres eoncernant la durée moyenne de la vie ont été établis en supposant qu'on laisse vivre l'animal jusqu'à sa fin naturelle. II le fallait ainsi pour que nous puissions établir une comparaison avec les autres animaux qui, eux, ne sont en général pas sacrifiés, et avec l'homme. En ce qui concerne la durée de l'alimentation lactée, nous l'avons rapportée aux conditions naturelles dans lesquelles vivent nos animaux.

Nous établirons plus loin quelles variations peuvent se produire dans la durée de l'alimentation lactée, suivant l'a ptitude laitière de la mère, et suivant le nombre de petits à la naissance.

Composition chimique du lait. - Nous n'en retiendrons que ce qui concerne les substances azotées et salines, aliment de construction pour le jeune.

Matières azotées. - Les matières azotées contenues dans le lait se divisent en matières protéiques (caséine, albumine, globuline), et en matières non protéiques qui se rencontrent à la dose de 1 à 2 pour 1.000 .

Le taux des matières protéiques varie a vec chaque espèce, et dans une même es pèce, il existe des oseillations individuelles. Il n'a pas une valeur constante durant toute la période de la lactation. Nous avons vu précédemment combien il était élevé quelques jours a vant la mise bas et même le jour de la naissance; puis ra pidement, le lendemain et les jours suivants, il décroît et atteint alors un chiffre sensiblement égal à celui du lait normal. Toutefois, il faut remarquer que la teneur en matières protéiques (et aussi en sels minéraux) décroît insensiblement jusqu'à la fin de la lactation. On observe quelques variations quotidiennes, mais jamais le taux de naissance n'est atteint.

Les matières protéiques (la caséine en particulier) sont indispensables au jeune pour se développér et grandir. Rappelons tout d'a bord les expériences de OsBorne et MENDEL, qui font ingérer à des rats des aliments ne contenant qu'une seule substance protéique. Les résultats furent les suivants : Pour que l'organisme puisse croître, il faut un minimum de 7 à $9 \%$ de matières protéiques ; pour une croissance normale, $18 \%$ sont nécessaires ; au-dessus de ce chiffre optimum, on observe une diminution de la croissance avec troubles nutritifs.

Donc, première conclusion : les matières protéiques sont indis pen- 
sables à l'organisme en voie de croissance, et la quantité optimum est de $18 \%$ environ.

OSBORNE et MENDEL montrèrent en outre que la nature de la substance protéique, et non pas seulement șa quantité, a une influence sur le rythme de la croissance. Pour cela, ils ont fait ingérer à des rats des matières protéiques isolées. Leurs expériences ont abouti aux conclusions suivantes :

La caséine et la lactabulmine du lait de vache donnent une croissance normale.

La gélatine, non seulement ne permet pas la croissance, mais encore fait dépérir l'animal.

La gliadine du blé ou du seigle, l'hordéine de l'orge empêchent l'animal de croître, mais lui garde son état d'équilibre.

Si l'on change l'alimentation, la croissance reprend rapide ; ̀̀ chacune de ces matières protéiques, il manque donc un principe essentiel, puisque, ingérées séparément, elles paralysent la croissance.

L'analyse chimique a révélé que la gélatine ne contient ni tryptophane, ni tyrosine, ni cystine ; l'hordéine et la gliadine sont dépourvues de glycocolle et de lysine. Champy et P. GLey ont montrésur des têtards que la croissance est impossible a vec des aliments sans lysine ; dès que cet élément est fourni, les animaux croissent ra pidement.

D'où deuxième conclusion : certains acides aminés sont indispensables à l'organisme en voie de croissance.

Ces expériences étant rappelées, étudions maintenant l'influence exercée sur la croissance par les matières protéiques contenues dans le lait.

Pour cette étude, nous diviserons en deux groupes les animaux dont nous nous sommes occupé.

11 existe en effet une opposition nette entre ceux qui naissent nus et a veugles et ceux qui, dès leur mise au monde, sont couverts et peuvent trotter et gambader.

Les premiers, isolés et soumis auxinfluences extérieures, seraient inca pables de grandir;ils ont done besoin d'une nourriture plus riche en matières protéiques et en sels minéraux que les autres, venant au monde plus forts et à un stade de développement plus avancé.

Il est nécessaire que, bien vite, les nouveau-nés soient capables d'acquérir une résistance suffisante et nécessaire pour un développement normal.

Lienfant occupe une place à part ; nous reviendrons sur ce sujet lorsque nous étudierons la composition du lait de femme.

Cette opposition qui existe à la naissance, au sein de l'ordre des mammifères, chez les animaux domestiques en particulier, doit inévitablement se retrouver dans la composition du seul aliment que les jeunes absorbent au début de leur vie : le lait. 
Le la pereau et le petit chien naissent, nous l'avons déjà vu, nus et a veugles, et leur poids de naissance se double assez rapidement; ce sont les deux animaux chez lesquels le poids augmente le plus vite.

ABDERHALDEN, qui fixe à 7 jours en moyenne le temps nécessaire au la pin pour doubler son poids, a, d'a près des analyses journalières, fixé à 10,29\% la teneur en matières protéiques du lait de la pine. Le taux de l'albumine est assez élevé $(2,07 \%)$. Dans les jours qui suivent le doublement du poids, le taux des matières protéiques tombe à $9 \%$ environ. Les recherches que nous avons faites, nous permettent d'évaluer le taux à un chiffre très sensiblement supérieur $(10,33 \%)$ et à 5 jours seulement le temps nécessaire au lapereau pour doubler son poids de naissance.

Les analyses du lait dans l'es pèce canine faites par ABDERHALDEN ont donné les résultats suivants :

La quantité de matières protéiques, très élevée le jour de la mise bas, est d'environ $9,20 \%$ (caséine, $6,10 \%$; albumine, $3,10 \%$ ); puis la baisse normale a lieu, si bien que, dans la période comprise entre la naissance et le jour où le jeune double son poids, on a le taux suivant : caséine, $4,84 \%$; albumine, $2,43 \%$. Après que le poids de naissance est doublé, les taux res pectifs deviennent : 4,42 \%-2,34\%. Des analyses de lait de chienne ont permis d'établir les moyennes suivantes indiquant la teneur totale en matières protéiques :

\section{$11,68 \%$ d'après VerNoIs et BeCQUEREL ; \\ $\mathbf{9 , 6 5 \%}$ » le Professeur Ch, Porcher ; \\ $7,29 \%$ 》 ABDERHALDEN.}

Il existe entre ces résultats une différence assez forte ; ceci provient de ce que les analyses ont été faites par chaque auteur à des époques fixes ; la moyenne obtenue ne représente donc pas la moyenne exacte de la teneur du lait en matières protéiques, depuis le début jusqu'à la fin de la lactation.

Nous avons pensé que le chiffre de $8,12 \%$ représentait assez exactement cette moyenne. Bref, les matières protéiques représentent environ ?a moitié de l'extrait sec ; leur taux peut donc être considéré comme élevé. ABDERHALDEN estime que cette valeủr doit être évaluée au $1 / 4$ seulement de l'extrait see; c'est vraiment un peu faible, et l'on comprend que les petits, nourris a vec un lait contenant des matières protéiques en aussi petite quantité, n'aient doublé leur poids qu'en 9 jours environ.

La différence avec le lait de la pine est assez forte ; et l'on a vu que le chiot mettait quelques jours de plus que le lapin pour doubler son poids initial.

Le chat ne fait pas partie de la catégorie des animaux naissant nus et a veugles, mais il établit une transition avec ceux qui, dès la mise bas, sont eapables de se mouvoir et y voient. Le chat, en effet (nous 
l'avons vu), naît couvert de poils mais a vec les yeux clos. Son énergie vitale n'est malgré cela guère plus considérable que chez le la pereau et le chiot ; mais il est moins sensible au froid par exemple et n'a pas besoin d'un lait ca pable de le faire grossir très ra pidement. ABDERHALDEN, pour qui le temps nécessaire au chat pour doubler son poids est de 9 jours $1 / 2$, fixe, d'a près les analyses des laits de 6 chattes, à $7 \%$ la teneur en matières protéiques $(3,71 \%$ pour la caséine, $3,29 \%$ pour l'albumine).

Nous a vons trou vé un taux légèrement supérieur (7,6 \% environ). D'après le Professeur Ch. Porcher, le lait de chatte contiendrait plus d'albumine que de caséine (près de deux fois plus).

Ainsi, le la pereau, le jeune chien, qui doublent en moins de 10 jours leur poids de naissance, reçoivent un lait très riche en matières protéiques. Si l'on compare à la valeur de l'extrait sec, c'est le lait de chienne qui a le taux le plus élevé en matières protéiques, puisqu'il en re présente plus de la moitié ; ensuite, viennent le lait de chatte $(50 \%$ environ ou un peu moins) et enfin celui de lapine (1/4 environ).

Il faut remarquer que, dans ces espèces, le taux d'albumine est très élevé ( $3 \%$ en moyenne); dans cette détermination, il y a aussi bien des divergences. Ainsi, d'a près le Professeur Porcher, ce pourcentage d'albumine serait plus élevé que celui de la caséine, chez la chienne et la chatte ; d'après ABDERHALDEN, ce serait le contraire ; mais les deux taux seraient très voisins.

Il est intéressant de comparer les laits des deux représentants domestiques de la famille des carnivores.

Il n'y a aucune différence sẹnsible dans le taux des matières protéiques, relativement à l'extrait sec. Les matières protéiques représentent généralement le $1 / 3$ de l'extrait sec ( $1 / 3 \mathrm{chez}$ la chienne, $\frac{1}{2,72}$ chez la chatte).

Au point de vue des proportions respectives de caséine et d'albumine, il n'y a pas une différence énorme, sauf que, chez le chat,l'albumine est en plus grande quantité que la caséine.

Nous a vons trouvé que, dans le lait de chienne, la caséine représente les $\frac{53}{100}$.des matières protéiques, et l'albumine $\frac{47}{100}$; différence minime qui permet même de dire que les quantités d'albumine et de caséine sont égales. Chez la chatte, les chiffres sont inverses a vec un écart de quelques centièmes (caséine : $\frac{44}{100}$, albumine $\frac{56}{100}$ ).

Ainsi, il y a uniformité dans le lait des carnivores, et la composition particulière du lait dans cette famille est une caractéristique importante.

Nous en arrivons maintenant au deuxième groupe, comprenant les 
animaux qui naissent couverts, qui y voient et qui peuvent marcher aussitôt la mise bas. A la limite de cette catégorie, nous trouvons le porc, qui, tout en arrivant au monde dans des conditions plus favorables que le la pin ôu le chien, naît malgré tout chétif et frêle ; sa mère lui est nécessaire pour le nourrir et pour le maintenir dans une douce chaleur.

Le lait de la truie est moins riche en albumine que les laits des femelles dont nous venons de nous occuper. ABDERHALDEN évalue à $1,36 \%$ la teneur du lait pour ce eomposant ; il a rencontré au cours de ses analyses des oscillations en plus ou en moins; il ne signale pas un taux su périeur à $1,65 \%$.

D'après le même auteur, la teneur moyenne en caséine serait de $3,39 \%$; comme pour tous les animaux, il y a une bassse sensible des matières protéiques le lendemain de la mise bas. Mais jusque vers le $13^{\mathrm{e}}$ ou le $14^{\mathrm{e}}$ jour, la quantité de matières protéiques reste sensiblement égale à ce qu'elle est le lendemain de la naissance. Bien après cette date, il y a une chute qui se poursuit jusqu'à la fin de la lactation.

Voici les moyennes récueillies par ABDERHALDEN, a près analyses des laits de trois truies :

Jusqu'au moment où le jeune double son poids de naissance (c.-à-d. à 14 jours)

Caséine albumine

$$
\left.\begin{array}{l}
3,57 \% \\
1,55 \%
\end{array}\right\} \text { Total : } 5,12 \%
$$

Après que le jeune a doublé son poids de naissance

$$
\left.\begin{array}{l}
3,16 \% \\
1,46 \%
\end{array}\right\}^{\prime} \text { Total : 4,62\%- }
$$

L'albumine baisse moins vite que la caséine.

Si l'on considère la totalité des matières protéiques, on consta te que leur valeur atteint environ $5 \%$ en moyenne (d'a près ABDERHALDEN : $4,75 \%$, et d'a près le Professeur Ch. Porcher, 5,92\%). Elle représente un peu moins du tiers de l'extrait sec ( $\frac{1}{7}$ exactement).

Dans l'espèce porcine, le taux de la caséine dans le lait est un peu plus de 2 fois $(2,35)$ celui de l'albumine.

Nous nous sommes demandé si, dans une même espèce, la croissance des petits était en rapport direct a vec la quantité des matières protéiques contenues dans le lait de la mère. Il n'en est rien; telle mère ayant un lait relativement riche en matières protéiques, aura des nouveau-nés dont le poids doublera moins vite que ceux d'une autre a yant un lait moins riche.

Ceci n'est pas seulement vrai pour le porc, mais pour tous les autres animaux et pour l'homme. Donc, dans une même es pèce, les variations individuelles du taux de matières protéiques sont sans action sur le rythme de la croissance.

Le cobaye met quelques jours de plus que le porcelet ponr doubler son poids de naissance. Mais, à ce moment-là, son alimentation lactée 
a cédé le pas de puis une semaine environ à l'alimentation de l'adulte, c'est-à-dire au foin, à la paille, aux bettera ves, à l'a voine. Nous n'insisterons pas assez sur ce fait que, si le cobaye arrive au monde a vec un développement très a vancé, il tète pendant quelques jours seulement. Aussi la sécrétion lactée de la mère cobaye se tarit très rapidement. Elle est assez abondante jusqu'à 6,8 jours a près la naissance, puis elle tombe très rapidement et dis paraît au bout de 16 à 18 jours.

A l'inverse de ce qui a lieu dans les autres espèces, la teneur en caséine est plus forte dans les jours qui suivent la naissance; elle passe par un maximum vers le $3^{\mathrm{e}}$ ou le $4^{\mathrm{e}}$ jour, puis une baisse a lieu qui aboutit à un minimum de $4,30 \%$ environ au $7 \mathrm{e}_{-} \mathrm{Se}$ jour ; une nouvelle augmentation se produit avec maximum supérieur au taux de croissance et jamais atteint jusque là, vers le $13^{\mathrm{e}}-14^{\mathrm{e}}$ jour, c'est-à-dire à l'époque où le jeune double son poids de naissance; puis, e'est de nouveau la descente progressive jusqu'à la fin de la lactation.

On ne trouve rien de semblable en ee qui concerne l'albumine, dont le taux décroit insensiblement à pariir de la naissance.

Les analyses de lait d'ABDERHALDEN' lui ont permis de fixer ainsi la teneur du lait de cobaye en matières protéiques :

\begin{tabular}{lcc} 
Caséine & $\begin{array}{c}\text { Jusqu'au moment où le jeune } \\
\text { double son poids de naissance } \\
\text { (c.-â-d. } 14 \text { jours) }\end{array}$ & $\begin{array}{c}\text { Dans les jours qui suivent } \\
\text { le doublement du poids }\end{array}$ \\
Albumine & $4,70 \%$ & $4,21 \%$ \\
$\quad$ Total & $0,55 \%$ & $0,42 \%$ \\
\hline $5,25 \%$ &
\end{tabular}

Dans cette es pèce, l'albumine est en très faible quantité, elle re présente seulement $1 / 10^{\mathrm{e}}$ environ de la totalité des matières protéiques.

Nous sommes maintenant en possession des analyses du lait de la pine, et il nous faut voir s'il y a chez les rongeurs uniformité dans les proportions, au point de vue des matières azotées.

Chez la lapine, comme chez le cobaye, les matières protéiques représentent le $\frac{1}{4}$ environ de l'extrait sec.

Les taux de caséine et d'albumine, comparés à la valeur totale des matières protéiques dans ces deux espèces sont les suivants :

$$
\text { Lapine }
$$

Cobaye

Caséine

Albumine

Ainsi, on peut dire que les teneurs respectives en caséine et en 
albumine sont les mêmes, comparativement à la valeur totale des matières protéiques, et comme moyenne on peut admettre :

$$
\begin{aligned}
& \text { Pour la caséine : } \frac{84}{100} \\
& \text { Pour l'albumine : } \frac{16}{100}
\end{aligned}
$$

Chez les rongeurs, même unité au point de vue des proportions.

Chez la brebis, le taux des matières protéiques, qui est de 6,58\% environ à la naissance de l'agneau, diminue progressivement jusque vers le $20^{\mathrm{e}}$ jour ; à partir de ce moment, il remonte pour passer par un maximum à l'époque où le jeune double son poids, $15^{\mathrm{e}}-17^{\mathrm{e}}$ jour, mais ici le chiffre de naissance n'est pas atteint (5,30\% environ), puis la descente recommence jusqu'à la fin de la lactation.

ABDERHALDEN, pour qui l'agneau met 14 jours à doubler son poids de naissance, évalue à 4,08 la valeur du taux de caséine, durant cette période, et a près, à $4,07 \%$; la différence est par conséquent minime. L'albumine passerait de $0,80 \%$ à $0,52 \%$.

La teneur moyenne en matières protéiques oscille autour de $5 \%$. Seuls, parmi nombre d'auteurs, Vernors et BeCQuerel donnent une valeur vraiment trop élevée : $6,97 \%$.

$\mathrm{Si}$ les chiffres concordent pour ce qui est de leur valeur absolue, il n'en est plus de même si l'on compare avec la valeur totale de l'extrait sec. ABDerHaLden évalue au 1/5 de l'extrait sec la teneur en protéines, alors que le Professeur Porcher l'évalue au 1/3. L'écart est assez considérable pour être signalé.

Nous admettons comme richesse du lait de brebis en matières protéiques : $5 \%$, et comme temps pour que l'agneau double son poids : 17 jours.

Le chevreau met quelques jours de plus que l'agneau pour doubler son poids de naissance : $22-23$ jours en moyenne. Or, nous avons trouvé que le lait de chèvre contient seulement $3,72 \%$ de matières protéiques, représentant la valeur globale de l'extrait sec.

ABDERHALDEN a longuement étudié la relation existant entre le développement du chevreau et la composition du lait de la mère.

Il estime le pourcentage des matières protéiques à $3,67 \%$ (caséine, $2,91 \%$; albumine, $0,76 \%$ ), dans la période s'étendant de la mise bas au $22{ }^{\mathrm{e}}$ jour, et à $3,14 \%$ dans les jours qui suivent.

(A suivre). 